\title{
Performance of Density Functional Theory Procedures for the Calculation of Proton-Exchange Barriers: Unusual Behavior of M06- Type Functionals
}

\author{
Bun Chan, ${ }^{*},+\ddagger$ Andrew T. B. Gilbert, ${ }^{\text {II }}$ Peter M. W. Gill, ${ }^{*}$, II and Leo Radom $*, \dagger, \ddagger$ \\ ${ }^{\dagger}$ School of Chemistry, University of Sydney, Sydney, NSW 2006, Australia \\ ${ }^{\ddagger}$ ARC Centre of Excellence for Free Radical Chemistry and Biotechnology and ${ }^{\mathbb{I I}}$ Research School of Chemistry, Australian National \\ University, Canberra, ACT 0200, Australia
}

\section{Supporting Information}

ABSTRACT: We have examined the performance of a variety of density functional theory procedures for the calculation of complexation energies and proton-exchange barriers, with a focus on the Minnesota-class of functionals that are generally highly robust and generally show good accuracy. A curious observation is that M05-type and M06-type methods show an atypical decrease in calculated barriers with increasing proportion of Hartree-Fock exchange. To obtain a clearer picture of the performance of the underlying components of M05-type and M06-type functionals, we have investigated the combination of MPW-type and PBE-type exchange and B95-type and PBE-type correlation procedures. We find that, for the extensive E3 test set, the general performance of the various hybrid-DFT procedures improves in the following order: PBE1-B95 $\rightarrow$ PBE1-PBE $\rightarrow$ MPW1-PBE $\rightarrow$ PW6-B95. As M05-type and M06-type procedures are related to PBE1-B95, it would be of interest to formulate and examine the general performance of an alternative Minnesota DFT method related to PW6-B95.

\section{INTRODUCTION}

Density functional theory (DFT) procedures ${ }^{1}$ are undeniably the workhorse for many computational chemists. This is due partly to their modest cost and, perhaps more importantly, to the level of accuracy (typically $\left.\sim 10-15 \mathrm{~kJ} \mathrm{~mol}^{-1}\right)^{2}$ that they are capable of achieving at this modest cost. Among the many contemporary DFT methods, the Minnesota family of functionals $^{3-7}$ have been found to perform well for many chemical applications. For instance, the M06-2X functional ${ }^{5 c}$ has achieved a weighted mean absolute deviation (weighted $\mathrm{MAD}$ ) from benchmark values of just $9.2 \mathrm{~kJ} \mathrm{~mol}^{-1}$ for the diverse GMTKN30 test set ${ }^{8}$ of 841 thermochemical quantities. ${ }^{2}$ In many of our own investigations, ${ }^{9}$ we also find that M06-type procedures, ${ }^{5}$ and their immediate predecessors, namely M05type functionals, ${ }^{4}$ are capable of yielding an accuracy that is exceeded only by some of the best double-hybrid DFT procedures and wave function-type composite protocols.

While the overall reliability of the Minnesota functionals has great practical utility for computational chemists, it would be unrealistic to consider these methods completely foolproof. For example, shortcomings have previously been noted associated with the integration grid, ${ }^{2,10}$ long-range dispersion, ${ }^{11}$ and an occasional relatively large dependence on the basis-set size. ${ }^{12}$ In fact, even the generally more robust higher-level wave functiontype procedures sometimes show remarkable failures for seemingly trivial problems. For instance, G4(MP2)-type composite procedures ${ }^{13}$ usually perform quite well when compared with the closely related but more rigorous G4 method, ${ }^{14}$ with typical MADs of $\sim 5 \mathrm{~kJ} \mathrm{~mol}^{-1}$ for both types of protocol. ${ }^{13,14}$ In a recent study, ${ }^{15}$ however, it was shown that, for complexation energies of water, ammonia and hydrogen fluoride clusters, and the associated proton-exchange barriers, G4(MP2)-type procedures yield substantially larger deviations from benchmark values (up to $\sim 30 \mathrm{~kJ} \mathrm{~mol}^{-1}$ ) than those for G4 ( $\sim 5 \mathrm{~kJ} \mathrm{~mol}^{-1}$ throughout).

The surprisingly poor performance of the generally robust G4(MP2)-type methods on these clusters raises the following question: how do the more economical but generally less accurate DFT procedures fare for this problem. We are particularly interested in the performance of the Minnesota functionals, due in part to their excellent record of being generally accurate and robust, as well as their widespread use as a result of their implementation in popular computational chemistry software packages such as Q-Chem ${ }^{16}$ and Gaussian. ${ }^{17}$ As we shall see in the discussion, the proton-exchange barriers that provide a challenge for G4(MP2)-type procedures also represent notable difficulties for some DFT procedures, including some of the Minnesota functionals. We hope that the results of the present study will shed light on strategies that may lead to the design of even better performing DFT procedures than the most accurate functionals that are currently available.

Received: June 10, 2014

Published: July 25, 2014 


\section{COMPUTATIONAL DETAILS}

Standard DFT calculations ${ }^{1}$ were carried out with Gaussian $09^{17}$ and Orca 2.8. ${ }^{18}$ For the systems examined in the present study, we have evaluated the dependence of the relative energies for several Minnesota functionals on the size of the integration grid, and we find that the use of the finest grid in Gaussian 09 (SuperFineGrid) instead of the default grid (FineGrid) leads to differences of less than $1 \mathrm{~kJ} \mathrm{~mol}^{-1}$. We have thus employed the FineGrid in all our calculations. It has previously been found that dispersion effects may be important for water clusters. ${ }^{2}$ As we shall see from Table 1, the mean deviations for the different DFT procedures are not uniformly positive or negative. Therefore, the inclusion of additional dispersion terms will not lead to a consistent improvement in

Table 1. Performance for the CEPX33 Set of Complexation Energies (CE) and Proton-Exchange Barriers (PX) for $\mathrm{H}_{2} \mathrm{O}$, $\mathrm{NH}_{3}$, and $\mathrm{HF}$ Clusters $\left(\mathrm{kJ} \mathrm{mol}^{-1}\right)$

\begin{tabular}{|c|c|c|c|c|c|c|}
\hline & \multicolumn{3}{|c|}{ mean absolute deviation } & \multicolumn{3}{|c|}{ mean deviation } \\
\hline & all & $\mathrm{CE}$ & PX & all & $\mathrm{CE}$ & PX \\
\hline \multirow[t]{2}{*}{ Hartree-Fock } & 68.7 & 41.4 & 110.6 & 68.7 & 41.4 & 110.6 \\
\hline & \multicolumn{6}{|c|}{ Nonhybrid Functionals } \\
\hline B-LYP & 18.0 & 13.7 & 24.6 & -2.8 & 11.4 & -24.6 \\
\hline PBE-PBE & 28.9 & 9.8 & 58.3 & -28.8 & -9.7 & -58.3 \\
\hline B-B95 & 21.1 & 22.9 & 18.2 & 6.7 & 22.9 & -18.2 \\
\hline PBE-B95 & 23.4 & 8.4 & 46.5 & -23.4 & -8.3 & -46.5 \\
\hline \multirow[t]{2}{*}{ VSXC } & 22.2 & 17.3 & 29.9 & 14.2 & 4.1 & 29.6 \\
\hline & \multicolumn{6}{|c|}{ B-LYP-Type Hybrid Functionals } \\
\hline B1-LYP & 9.2 & 10.4 & 7.3 & 2.9 & 7.9 & -5.0 \\
\hline BH\&H-LYP & 9.3 & 6.7 & 13.2 & 5.1 & 2.0 & 9.8 \\
\hline B3-LYP & 11.3 & 9.3 & 14.5 & -2.4 & 5.5 & -14.5 \\
\hline \multirow[t]{2}{*}{ CAM-B3-LYP } & 19.4 & 12.0 & 30.7 & -17.6 & -9.1 & -30.7 \\
\hline & \multicolumn{6}{|c|}{ PBE-Type Hybrid Functionals } \\
\hline PBE1-PBE & 15.6 & 5.8 & 30.8 & -14.8 & -4.3 & -30.8 \\
\hline LC- $\omega$ PBE & 11.6 & 8.9 & 15.6 & -1.1 & 7.8 & -14.7 \\
\hline \multirow[t]{2}{*}{ HSEh1-PBE } & 17.3 & 7.7 & 32.1 & -16.9 & -7.0 & -32.1 \\
\hline & \multicolumn{6}{|c|}{ B97-Type Hybrid Functionals } \\
\hline B98 & 8.9 & 4.3 & 15.8 & -5.0 & 2.0 & -15.8 \\
\hline BMK & 11.0 & 12.2 & 9.3 & 11.0 & 12.1 & 9.3 \\
\hline \multirow[t]{2}{*}{$\omega \mathrm{B} 97 \mathrm{X}$} & 15.3 & 15.2 & 15.4 & -15.3 & -15.2 & -15.4 \\
\hline & \multicolumn{6}{|c|}{ Mixed-PBE-B95 Hybrid Functional } \\
\hline \multirow[t]{2}{*}{$\mathrm{PBE}_{1-B 95^{a}}$} & 10.9 & 5.4 & 19.4 & -9.7 & -3.4 & -19.4 \\
\hline & \multicolumn{6}{|c|}{ Pre-M05 Minnesota Functionals } \\
\hline MPW1-B95 & 7.4 & 7.5 & 7.2 & 1.3 & 6.3 & -6.3 \\
\hline MPW-B1K & 6.3 & 6.7 & 5.8 & 5.1 & 5.8 & 4.0 \\
\hline PW6-B95 & 6.0 & 6.3 & 5.4 & 1.3 & 4.5 & -3.6 \\
\hline \multirow[t]{2}{*}{ PW-B6K } & 5.6 & 4.8 & 6.9 & 0.0 & -1.9 & 3.0 \\
\hline & \multicolumn{6}{|c|}{ M05-Type and M06-Type Functionals } \\
\hline M05 & 7.2 & 5.5 & 9.8 & -4.7 & -5.4 & -3.6 \\
\hline M05-2X & 18.9 & 7.7 & 36.3 & -17.4 & -5.1 & -36.3 \\
\hline M06-L & 4.5 & 4.0 & 5.2 & 3.4 & 2.3 & 5.0 \\
\hline M06 & 5.0 & 3.7 & 7.1 & -0.5 & -1.0 & 0.2 \\
\hline M06-2X & 15.7 & 7.2 & 28.9 & -14.9 & -5.8 & -28.9 \\
\hline \multirow[t]{2}{*}{ M06-HF } & 35.4 & 14.0 & 68.4 & -32.9 & -9.8 & -68.4 \\
\hline & \multicolumn{6}{|c|}{ Post-M06-Type Minnesota Functionals } \\
\hline M08-HX & 7.5 & 7.8 & 7.0 & -6.3 & -7.8 & -4.1 \\
\hline M08-SO & 6.1 & 6.7 & 5.1 & -5.8 & -6.7 & -4.4 \\
\hline M11-L & 15.1 & 13.5 & 17.6 & 14.6 & 13.5 & 16.3 \\
\hline M11 & 14.6 & 7.1 & 26.1 & -12.8 & -4.1 & -26.1 \\
\hline
\end{tabular}

${ }^{a}$ Introduced in the present study and containing 25\% Hartree-Fock exchange, $75 \%$ PBE exchange and 100\% B95 correlation. the agreement with benchmark values for all methods, and we have not applied such corrections in the present study.

Geometries, zero-point vibrational energies, thermal corrections for $298 \mathrm{~K}$ enthalpies, and benchmark total energies that are used in the present investigation were taken from previous studies. Specifically, those for the water, ammonia and hydrogen fluoride clusters were obtained from ref 15 , while those for species in the E3 test set ${ }^{14,19}$ (which includes all the data from the G2/97 test $\operatorname{set}^{20}$ ) were taken from ref 19. For main-group elements, we used two triple- $\zeta$-valence Pople-type basis sets for single-point energy calculations. Specifically, for the calculation of complexation energies and proton-exchange barriers of the $\mathrm{H}_{2} \mathrm{O}, \mathrm{NH}_{3}$, and $\mathrm{HF}$ clusters, we used the 6$311+\mathrm{G}(3 \mathrm{df}, 2 \mathrm{p})$ basis set. For the evaluation of energies in the E3 and G2/97 sets, we used the closely related 6-311+ $+\mathrm{G}(3 \mathrm{df}, 3 \mathrm{pd})$ basis set, as in another recent study. ${ }^{21}$ A number of species in the E3 set include Pd and Ru. In accordance with ref 21, the def2-TZVP basis set, together with the matching effective core potential, ${ }^{22}$ was employed for these elements.

All relative energies are reported in $\mathrm{kJ} \mathrm{mol}^{-1}$. Deviations are defined as "calculated value minus benchmark value". In a number of cases where we wish to explicitly designate either the exchange or the correlation component of a particular functional, we use the lowercase suffixes " $x$ " and " $c$ ", respectively.

\section{RESULTS AND DISCUSSION}

Overview of M05-Type and M06-Type DFT Procedures. As we shall see, the family of M05-type and M06-type functionals is a major focus of the present study. It is thus instructive to provide a brief overview of the main features of these methods.

The M05 class of DFT functionals shares the following ingredients:

1. an exchange part based on the PBE functional, ${ }^{23}$

2. a kinetic energy density $(\tau)$ enhancement factor in the exchange component, expanded as a polynomial,

3. a proportion of Hartree-Fock exchange,

4. a correlation functional based on the correlation component of B97 (B97c), ${ }^{24}$ where the opposite-spin and same-spin correlations are treated with two separate polynomials, each containing a nonlinear parameter and a number of linear parameters,

5. a term to correct for the same-spin self-interaction error,

6. fitting of a number of parameters in this "hybrid- $\tau$-PBExB97c" functional to an extensive training set of thermochemical properties. There are 12 parameters in the $\tau$-enhancement factor, one fixed to unity in order to recover the correct uniform-electron-gas (UEG) limit, five parameters for the opposite-spin correlation (one fixed to unity to satisfy the UEG limit), and five for the same-spin correlation (likewise, one fixed to unity). Together with the proportion of Hartree-Fock exchange, these yield a total of 23 linear parameters, of which 20 are adjustable and fitted to the training set.

The family of M06-type functionals is based on the M05-type functionals, with the following differences:

1. a proportion of the M05 exchange-correlation is replaced by the VSXC functional, ${ }^{25}$ which itself contains a number of adjustable parameters,

2. the addition of the VSXC terms leads to a total of 38 parameters, of which 35 are independently fitted.

The Performance of DFT Procedures for Complexation Energies and Proton-Exchange Barrier Heights for 
$\mathrm{H}_{2} \mathrm{O}, \mathrm{NH}_{3}$, and HF Clusters. Figure 1 shows illustrative examples of (a) a complex and (b) a transition structure from

(a)

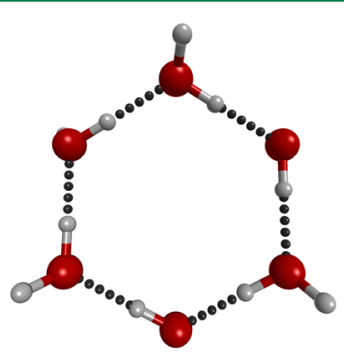

Figure 1. (a) Six-water complex and (b) the corresponding transition structure for proton-exchange.

ref 15 that are used in the present study. The complete set of data contains 20 complexation energies and 13 barrier heights, obtained with the high-level W1-F12 procedure, ${ }^{26}$ for clusters and transition structures for proton exchange that include 2-6 water, 2-6 ammonia, or 2-6 hydrogen fluoride molecules. We will denote this set of data as the complexation energy (CE) and proton-exchange (PX) 33 set (CEPX33).

Functionals Examined. While there are a large number of DFT procedures available in widely used computational chemistry software packages, we note that many functionals can be loosely grouped into a relatively small number of categories. Therefore, we have investigated the performance of a small but representative selection of methods, with the first group of procedures based on B-LYP, ${ }^{27}$ the second class containing methods derived from the $\mathrm{PBE}$ exchange and correlation functionals, ${ }^{23}$ and the third class consisting of procedures related to $\mathrm{B} 95$ or $\mathrm{B} 97$. $^{24,28,29}$

At the simplest level, we have examined the nonhybrid functionals B-LYP, PBE-PBE, B-B95, PBE-B95, and VSXC. The PBE-B95 and VSXC procedures are included in particular as they are related to M05-type and M06-type functionals (B95 included in B-B95 and PBE-B95 is related to the B97c-type functional in the M05-type and M06-type methods). ${ }^{4,5}$

A range of related hybrid-DFT procedures have also been investigated in the present study. They are B-LYP-type functionals B1-LYP, ${ }^{30}$ BH\&H-LYP, ${ }^{17,31} \mathrm{~B} 3-\mathrm{LYP},{ }^{32}$ and CAMB3-LYP, ${ }^{33}$ PBE-type functionals PBE1-PBE, ${ }^{34}$ LC- $\omega$ PBE, ${ }^{35}$ and HSEh1-PBE, ${ }^{36}$ and B97-type functionals $\mathrm{B} 98,{ }^{37} \mathrm{BMK},{ }^{38}$ and $\omega \mathrm{B} 97 \mathrm{X} .{ }^{39}$ We have also included a hybrid functional based on the PBE exchange with 25\% Hartree-Fock exchange and B95 correlation. This procedure is termed PBE1-B95 in the present study. The inclusion of this method is again motivated by its close relationship with M05-type and M06-type functionals.

Finally, we have investigated various DFT procedures due to Truhlar and co-workers. These include several methods (MPW1-B95, ${ }^{3 \mathrm{a}}$ MPW-B1K, ${ }^{3 \mathrm{a}}$ PW6-B95, ${ }^{3 \mathrm{~b}}$ and PW-B6K ${ }^{3 \mathrm{~b}}$ ) that generally perform quite well and preceded the M05-type and M06-type procedures (M05, ${ }^{4 \mathrm{a}} \mathrm{M} 05-2 \mathrm{X},{ }^{4 \mathrm{~b}} \mathrm{M} 06-\mathrm{L},{ }^{5 \mathrm{a}} \mathrm{M} 06,{ }^{5 \mathrm{c}}$ $\mathrm{M} 06-2 \mathrm{X},{ }^{5 \mathrm{c}}$ and $\left.\mathrm{M} 06-\mathrm{HF}^{5 \mathrm{~b}}\right)$, as well as several functionals that succeeded M06-type procedures, specifically M08-HX, ${ }^{6}$ M08SO, ${ }^{6}$ M11-L, ${ }^{7 b}$ and M11. ${ }^{7 a}$ For comparison, we have also examined the performance of Hartree-Fock for the CEPX33 set.

Hartree-Fock, GGA, and Hybrid-GGA Functionals. The mean absolute deviations (MADs) and mean deviations (MDs) from the benchmark W1-F12 results for the Hartree-Fock and various DFT procedures for the CEPX33 set are shown in
Table 1. We first note the large MADs $\left(68.7 \mathrm{~kJ} \mathrm{~mol}^{-1}\right.$ overall) for Hartree-Fock, which are due mostly to its systematic overestimation of both the complexation energies and (especially) the barrier heights, with $\mathrm{MD}$ values that are positive and of the same magnitude as those for the MADs. The observation that deviations for the barriers are larger than those for complexation energies is in accord with previous findings. ${ }^{15}$

The nonhybrid functionals yield much improved performance compared with Hartree-Fock, with overall MADs of $\sim 20-30 \mathrm{~kJ} \mathrm{~mol}^{-1}$. We again find that the deviations for barriers are, in general, larger than those for complexation energies. We can see from the MDs that, while B-LYP, B-B95, and VSXC are like Hartree-Fock in typically overestimating complexation energies, $\mathrm{PBE}-\mathrm{PBE}$ and $\mathrm{PBE}-\mathrm{B} 95$ tend to underestimate these values. The MD values for the B-LYP, PBE-PBE, B-B95 and PBE-B95 barrier heights are negative, while that for VSXC is positive. Among the five functionals in this group, only PBEPBE and PBE-B95 exhibit the same sign for the MDs for complexation energies and barrier heights. We note that the performance of PBE-B95 is closer to that for PBE-PBE than to B-B95. Overall, however, we find that there is little consistency regarding systematic behavior among the different nonhybrid DFT methods.

We now turn our attention to the various groups of nonMinnesota hybrid functionals from B1-LYP to PBE1-B95 in Table 1. In general, they outperform their nonhybrid counterparts, with overall MADs of $\sim 10-20 \mathrm{~kJ} \mathrm{~mol}^{-1}$. We note that the use of range-separation (CAM-B3-LYP, HSEh1$\mathrm{PBE}$, and $\omega \mathrm{B} 97 \mathrm{X})$ often leads to a deterioration in performance for the CEPX33 set of systems. It is noteworthy that the B-LYP, B1-LYP, and BH\&H-LYP functionals differ only in the proportion of Hartree-Fock exchange [B-LYP $(0 \%)<$ B1LYP $(25 \%)<$ BH\&H-LYP $(50 \%)]$, and we can see that increasing the amount of Hartree-Fock exchange leads to a less negative $\mathrm{MD}$ for the barriers (Table 1, Figure 2). This is consistent with the general view that increasing the proportion of Hartree-Fock exchange would tend to give larger barriers. $^{1,40}$

As an aside, the results in Table 1 suggest only a relatively small degree of "multireference" character for the systems in the CEPX33 set, using the B1 diagnostic ${ }^{41}$ as an indicator. Thus, a comparison of the MDs for B-LYP and B1-LYP points to average $\mathrm{B} 1$ values of $3.5 \mathrm{~kJ} \mathrm{~mol}^{-1}$ for complexation energies and

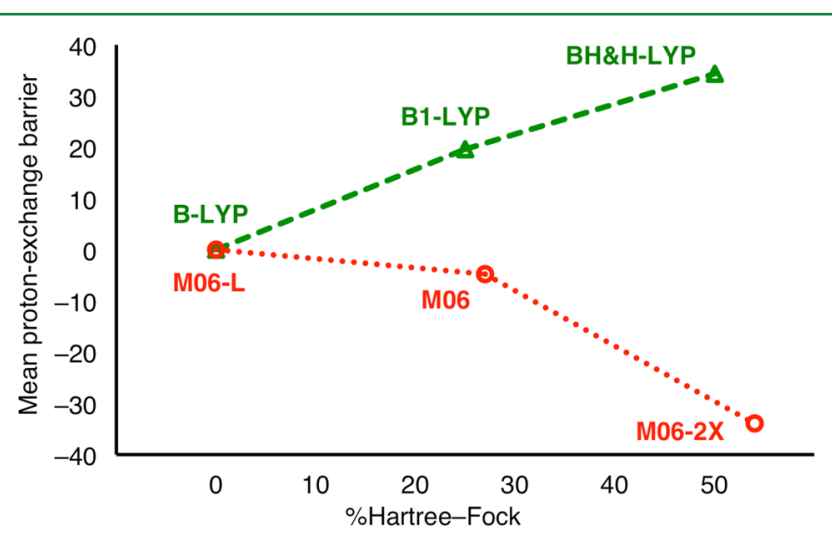

Figure 2. Mean proton-exchange barriers $\left(\mathrm{kJ} \mathrm{mol}^{-1}\right)$ for B-LYP-type functionals (B-LYP, B1-LYP, and BH\&H-LYP relative to the value for $\mathrm{B}-\mathrm{LYP}$ ) and M06-type functionals (M06-L, M06, and M06-2X relative to that for M06-L), showing contrasting behavior as the proportion of Hartree-Fock exchange is increased. 
$19.6 \mathrm{~kJ} \mathrm{~mol}^{-1}$ for barrier heights, which are considerably smaller than the threshold of $10 \mathrm{kcal} \mathrm{mol}^{-1}\left(41.8 \mathrm{~kJ} \mathrm{~mol}^{-1}\right)$ for significant multireference character.

Minnesota Functionals. The four hybrid functionals of Zhao and Truhlar that preceded M05 (MPW1-B95, MPWB1K, PW6-B95, PW-B6K) perform well for the CEPX33 set, and this is in accord with their good performance in general. ${ }^{2}$ We note that, when compared with other functionals so far discussed, these methods show a smaller tendency for overestimation or underestimation of the complexation energies and barriers. Thus, the magnitudes of their MDs are less than $10 \mathrm{~kJ} \mathrm{~mol}^{-1}$ throughout. In contrast to the generally mild deviations for these pre-M05 functionals, there is a considerable variation in the performance of the M05-type and M06-type procedures for the CEPX33 set. The M05, M06-L, and M06 procedures perform quite well (overall MADs $\sim 5-$ $10 \mathrm{~kJ} \mathrm{~mol}^{-1}$ ), but M05-2X, M06-2X, and (especially) M06-HF have significantly larger overall MADs (up to $35.4 \mathrm{~kJ} \mathrm{~mol}^{-1}$ for M06-HF). On the other hand, in a recent study of protonexchange barriers for a different set of systems, ${ }^{42}$ the M05-type and M06-type methods perform quite well. However, all functionals examined in that study have MADs from benchmark values of less than $5 \mathrm{~kJ} \mathrm{~mol}^{-1}$ (e.g., $3.8 \mathrm{~kJ} \mathrm{~mol}^{-1}$ for B3LYP). We have briefly investigated some of the systems in that set that are closely related to those in the CEPX33 set with BLYP, B1-LYP, and BH\&H-LYP and find that their performances against our W1X-2 ${ }^{43}$ benchmarks do not differ significantly, with MADs of 4.6 (B-LYP), 3.4 (B1-LYP), and 1.8 (BH\&H-LYP) $\mathrm{kJ} \mathrm{mol}^{-1}$. This also implies an average B1 diagnostic value of just $1.2 \mathrm{~kJ} \mathrm{~mol}^{-1}$.

We can see that the MD value for barriers for M05-2X is significantly more negative than that for M05. Likewise, the MDs for the barriers become more negative for M06-type functionals along the sequence M06-L $\rightarrow$ M06 $\rightarrow$ M06-2X $\rightarrow$ M06-HF (Figure 2). These trends are curious when we consider that M05-2X doubles the proportion of Hartree-Fock exchange when compared with that in M05, and that the amount of Hartree-Fock exchange for M06-type procedures increases in the order M06-L $\rightarrow$ M06 $\rightarrow$ M06-2X $\rightarrow$ M06-HF. Thus, the correlation between these barrier heights and the proportion of Hartree-Fock exchange in M05-type and M06type functionals appears to be the opposite of that for typical DFT procedures such as B-LYP versus B1-LYP versus BH\&HLYP. We also note that, for a set of pericyclic reactions (the BHPERI set) within the GMTKN30 set, M06-2X generally yields smaller barriers than M06, but for other reaction types (the BH76 set of hydrogen-atom transfer, heavy-atom transfer, nucleophilic substitution, unimolecular and association reactions), M06-2X typically gives larger barriers than $\mathrm{M}^{2} 6^{2} \mathrm{We}$ stress, however, that the proportions of Hartree-Fock exchange in M05-type and M06-type functionals are closely linked to the rest of the parameters, and these are different for each procedure. Therefore, the effect of increasing this proportion on the relative energies for these methods may not be trivially generalizable, but the curious observation nonetheless deserves further exploration.

Among the four successors to the M06-type functionals, M08-HX and M08-SO both perform quite well, with MADs that are comparable to those for the procedures that are found to be the most precise for the CEPX33 set (e.g., PW6-B95 and PW-B6K). In addition, the magnitudes of the MDs for M08HX and M08-SO are among the smaller values for the DFT methods examined, which is indicative of comparatively mild systematic deviations for these systems. On the other hand, M11-L and M11 have MAD and MD magnitudes that are larger and comparable to those for the other typical hybrid functionals rather than the best-performing ones.

The Dependence on the Amount of Hartree-Fock Exchange. In the previous section, we saw that the mean deviations in the barriers obtained with M05-type and M06type functionals become more negative with an increasing amount of Hartree-Fock exchange, in contrast to the behavior of typical DFT methods (Figure 2). In an attempt to identify the features of the M06-type functionals that might be responsible for the peculiar proton-exchange barriers, we have further broken down the barriers for six DFT procedures, namely B-LYP, B1-LYP, BH\&H-LYP, M06-L, M06, and M06$2 \mathrm{X}$, into three components.

The first three functionals represent typical DFT methods with an increasing proportion of Hartree-Fock exchange of $0 \%$ (B-LYP), 25\% (B1-LYP), and 50\% (BH\&H-LYP), ${ }^{44}$ while the latter three are M06-type procedures that contain 0\% (M06-L), 27\% (M06), and 54\% (M06-2X) Hartree-Fock exchange. Two of the energy components for the barriers are (1) classical interactions associated with nuclear-nuclear repulsion, nuclear-electron attraction and electron-electron repulsion and (2) Fock exchange, with the sum of these two terms being the Hartree-Fock component of the total hybrid-DFT energy. The third is the exchange-correlation energy associated with the density functional. To enable a straightforward observation of the variations in the components of the barriers, we have listed energies relative to the values for the zero Hartree-Fock exchange variants, i.e., B-LYP for B1-LYP and BH\&H-LYP and M06-L for M06 and M06-2X (Table 2). Thus, to examine the general trends for the two types of DFT procedures, comparisons can be made between B1-LYP and M06 and between BH\&H-LYP and M06-2X.

It can be seen that an increase in the proportion of HartreeFock exchange leads to a more negative energy contribution from the classical components for both B-LYP-type as well as M06-type procedures. The magnitudes of these changes are not too dissimilar between these two classes of functionals. Likewise, B-LYP-type and M06-type methods behave similarly for the Fock-exchange component as the amount of HartreeFock exchange is increased. Thus, in both cases, an increase in the proportion of Hartree-Fock exchange contributes to larger barriers, as one would expect. The magnitudes of these changes are again reasonably comparable for the two classes of procedures.

For B-LYP-type functionals, the effect of increasing the proportion of Hartree-Fock exchange on the DFT-exchangecorrelation component of the barriers is to make them more positive, with the exception of the two-water cluster for which the opposite trend is observed. On the other hand, the M06type exchange-correlation contributions to the barriers become more negative as the proportion of Hartree-Fock exchange increases in all cases, most notably for the two-water cluster. Given the similarities in behavior between B-LYP and M06-2X for the classical and Fock-exchange terms, the difference in behavior for the DFT exchange-correlation component appears to be the main reason for the opposite trends observed in the overall barriers calculated by B-LYP-type and M06-type procedures as the proportion of Hartree-Fock exchange is increased.

General Performance of Different Combinations of Exchange and Correlation Functionals Related to 
Table 2. Relative Energy Variations $\left(\mathrm{kJ} \mathrm{mol}^{-1}\right)$ for B-LYPType and M06-Type DFT Procedures for the Contributions of Hybrid-DFT Components to the Total Proton-Exchange Barriers for Clusters with Two to Six Water Molecules

\begin{tabular}{|c|c|c|c|c|c|c|}
\hline & B-LYP & B1-LYP & BH\&H-LYP & M06-L & M06 & M06-2X \\
\hline & $0 \% \mathrm{HF}$ & $25 \% \mathrm{HF}$ & $50 \% \mathrm{HF}$ & $0 \% \mathrm{HF}$ & $27 \% \mathrm{HF}$ & $54 \% \mathrm{HF}$ \\
\hline & \multicolumn{6}{|c|}{ Classical Component } \\
\hline 2 & 0.0 & -9.0 & -13.0 & 0.0 & -1.1 & -7.9 \\
\hline 3 & 0.0 & -14.5 & -23.2 & 0.0 & -10.0 & -21.5 \\
\hline 4 & 0.0 & -18.2 & -29.6 & 0.0 & -15.9 & -35.3 \\
\hline 5 & 0.0 & -21.5 & -35.1 & 0.0 & -19.0 & -44.5 \\
\hline \multirow[t]{2}{*}{6} & 0.0 & -24.3 & -39.5 & 0.0 & -18.7 & -52.6 \\
\hline & \multicolumn{6}{|c|}{ Fock-Exchange Component } \\
\hline 2 & 0.0 & 47.9 & 94.1 & 0.0 & 51.6 & 106.3 \\
\hline 3 & 0.0 & 22.4 & 44.2 & 0.0 & 25.2 & 56.3 \\
\hline 4 & 0.0 & 11.8 & 23.0 & 0.0 & 15.3 & 41.0 \\
\hline 5 & 0.0 & 8.1 & 15.5 & 0.0 & 11.8 & 37.4 \\
\hline \multirow[t]{2}{*}{6} & 0.0 & 4.9 & 8.5 & 0.0 & 7.1 & 32.8 \\
\hline & \multicolumn{6}{|c|}{ DFT-Exchange-Correlation Component } \\
\hline 2 & 0.0 & -16.5 & -39.5 & 0.0 & -56.2 & -109.3 \\
\hline 3 & 0.0 & 10.0 & 10.8 & 0.0 & -24.8 & -62.1 \\
\hline 4 & 0.0 & 24.6 & 38.0 & 0.0 & -11.7 & -45.3 \\
\hline 5 & 0.0 & 34.1 & 55.0 & 0.0 & -6.6 & -43.1 \\
\hline \multirow[t]{2}{*}{6} & 0.0 & 42.6 & 70.3 & 0.0 & -7.4 & -42.6 \\
\hline & \multicolumn{6}{|c|}{ Total } \\
\hline 2 & 0.0 & 22.4 & 41.6 & 0.0 & -5.7 & -10.9 \\
\hline 3 & 0.0 & 17.9 & 31.8 & 0.0 & -9.5 & -27.4 \\
\hline 4 & 0.0 & 18.2 & 31.4 & 0.0 & -12.2 & -39.6 \\
\hline 5 & 0.0 & 20.6 & 35.3 & 0.0 & -13.8 & -50.2 \\
\hline 6 & 0.0 & 23.1 & 39.3 & 0.0 & -19.0 & -62.4 \\
\hline
\end{tabular}

Minnesota DFT Procedures. As outlined earlier, M05-type functionals are essentially hybrid- $\tau$-PBEx-B97c methods with a number of fitted parameters, while M06-type procedures additionally incorporate terms from the VSXC functional. While the peculiar trends of M06-type (and M05-type) procedures for the proton-exchange barriers in the CEPX33 set may potentially be a cause for caution more generally and it is desirable to rationalize this behavior, it would be difficult to unambiguously determine the cause due to the large number of coupled parameters.

To this end, it is of interest to elucidate the performance of related and simpler procedures. We recall that the pre-M05 Minnesota functionals (MPW1-B95, MPW-B1K, PW6-B95, and PW-B6K) perform quite well for the CEPX33 set (Table 1). In addition, the PW6-B95 procedure has been shown to perform almost as well as M06-2X for the GMTKN30 test set. ${ }^{2}$ We note that these early Minnesota functionals contain relatively few parameters that are fitted to a training set (up to six for PW6-B95 and PW-B6K). Thus, we deem it useful to attempt to unravel the features that may have led to their good performance with only very mild parametrization.

With these considerations in mind, we now proceed to examine the performance of several Minnesota functionals more generally and make comparison with a number of relevant other DFT procedures. We use the moderately sized G2/97 test set and its subsets for our preliminary investigation. The results are shown in Table 3. For the M05-type and M06-type methods, the performance generally improves in the order M05 $\rightarrow$ M06 $\rightarrow$ M05-2X $\rightarrow$ M06-2X, with overall MADs of 15.8, $14.3,14.0$, and $10.8 \mathrm{~kJ} \mathrm{~mol}^{-1}$, respectively.
Table 3. Mean Absolute Deviations $\left(\mathrm{kJ} \mathrm{mol}^{-1}\right)$ of the Various DFT Procedures from Benchmark Values for the G2/97 Test Set and Its Subsets ${ }^{a}$

\begin{tabular}{|c|c|c|c|c|}
\hline & \multicolumn{4}{|c|}{ DFT procedure } \\
\hline & PBE1-B95 & PBE1-PBE & MPW1-B95 & PW6-B95 \\
\hline $\mathrm{G} 2 / 97$ & 27.9 & 18.3 & 15.4 & 11.1 \\
\hline$\Delta H_{\mathrm{f}}$ & 42.1 & 20.0 & 16.5 & 10.3 \\
\hline IE & 16.9 & 18.9 & 15.9 & 14.0 \\
\hline EA & 10.4 & 15.2 & 13.1 & 10.0 \\
\hline \multirow[t]{3}{*}{ PA } & 7.1 & 4.6 & 4.9 & 3.9 \\
\hline & \multicolumn{4}{|c|}{ DFT procedure } \\
\hline & M05 & M06 & M05-2X & M06-2X \\
\hline $\mathrm{G} 2 / 97$ & 15.8 & 14.3 & 14.0 & 10.8 \\
\hline$\Delta H_{\mathrm{f}}$ & 14.8 & 15.8 & 13.7 & 9.6 \\
\hline $\mathrm{IE}$ & 20.2 & 15.5 & 15.8 & 13.0 \\
\hline EA & 12.9 & 9.7 & 13.1 & 11.1 \\
\hline $\mathrm{PA}$ & 8.6 & 8.3 & 6.6 & 8.8 \\
\hline \multicolumn{5}{|c|}{$\begin{array}{l}{ }^{a} \Delta H_{\mathrm{f}}=\text { heat of formation, } \mathrm{IE}=\text { ionization energy, } \mathrm{EA}=\text { electror } \\
\text { affinity, and } \mathrm{PA}=\text { proton affinity. }\end{array}$} \\
\hline
\end{tabular}

For the other procedures, we first note that PW6-B95 has MAD values that are quite small and comparable to those for M06-2X. The performance for the G2/97 test set improves in the order PBE1-B95 (overall MAD $=27.9 \mathrm{~kJ} \mathrm{~mol}^{-1}$ ) $\rightarrow$ PBE1PBE (18.3) $\rightarrow$ MPW1-B95 (15.4) $\rightarrow$ PW6-B95 (11.1). This suggests that the combination of MPW-type exchange functionals and B95-type correlation functionals are capable of producing quite accurate results. On the other hand, the pairing of $\mathrm{PBE}$ and $\mathrm{B} 95$ in a DFT procedure seems to give rather disappointing results but mainly for heats of formation. We can see that PBE1-PBE gives reasonable results, but its performance for the G2/97 set is not as good as those for the two hybrid-MPW-B95-type procedures.

In order to further generalize the above observations regarding the combination of exchange (MPW or PBEx) and correlation (B95 or PBEc) functionals, we have assessed five DFT procedures PW6-B95, MPW1-B95, MPW1-PBE, PBE1PBE, and PBE1-B95 with the more extensive E3 test set. ${ }^{19}$ This set contains data not only for fundamental thermochemical properties but also for commonplace chemical reactions (e.g., DBH24, PR8, and Grubbs), as well as specially challenging cases (MB08) for computational chemistry procedures. The results are shown in Table 4, together with those for B3-LYP and B3-PW91 from ref 21 for comparison. We highlight that the results for the E3 set are, in general, qualitatively similar to the observations for G2/97. In particular, hybrid-MPW-B95type procedures perform somewhat better than PBE1-PBE (and MPW1-PBE), which in turn are overall more accurate than PBE1-B95. However, we again emphasize that the difference in the performance is mainly due to large differences in performance for heats of formation (W4/08, G2/97' $\Delta H_{\mathrm{f}}$, and $\mathrm{G} 3 / 99^{\prime}$ ).

Implications and Recommendations. We recall that M05-type procedures are based on $\tau$-hybrid-PBEx-B97c, and such a functional form is also a major ingredient of M06-type procedures. The observations regarding hybrid-PBE-B95-type procedures (Table 3 and Table 4) suggest that such a choice of underlying components in M05-type and M06-type methods may not be entirely optimum. We note that M08-type functionals are based on PBE for both the exchange and the correlation components, which may put M08-type methods on 
Table 4. Mean Absolute Deviations $\left(\mathrm{kJ} \mathrm{mol}^{-1}\right)$ of the Various DFT Procedures from Benchmark Values for the E3 Test Set and Its Subsets ${ }^{a}$

\begin{tabular}{|c|c|c|c|c|c|c|c|}
\hline & $\begin{array}{l}\text { PW6- } \\
\text { B95 }\end{array}$ & $\begin{array}{l}\text { MPW1- } \\
\text { B95 }\end{array}$ & $\begin{array}{c}\text { MPW1- } \\
\text { PBE }\end{array}$ & $\begin{array}{l}\text { PBE1- } \\
\text { PBE }\end{array}$ & $\begin{array}{l}\text { PBE1- } \\
\text { B95 }\end{array}$ & $\begin{array}{l}\text { B3- } \\
\text { LYP }\end{array}$ & $\begin{array}{c}\text { B3- } \\
\text { PW91 }\end{array}$ \\
\hline E3 & 12.9 & 17.0 & 19.3 & 22.7 & 30.1 & 17.1 & 19.7 \\
\hline & \multicolumn{7}{|c|}{ E0 Subset } \\
\hline All & 9.1 & 9.9 & 14.2 & 13.1 & 20.6 & 12.0 & 12.9 \\
\hline W4/08 & 10.5 & 12.0 & 17.2 & 15.0 & 26.5 & 13.4 & 14.6 \\
\hline $\mathrm{DBH} 24$ & 11.5 & 10.2 & 13.9 & 15.4 & 15.6 & 15.9 & 17.4 \\
\hline HB16 & 1.8 & 1.9 & 3.4 & 4.6 & 3.0 & 2.9 & 2.4 \\
\hline \multirow[t]{2}{*}{ WI9/04 } & 0.7 & 1.0 & 1.5 & 1.1 & 0.7 & 2.2 & 1.9 \\
\hline & \multicolumn{7}{|c|}{ G2/97' Subset } \\
\hline All & 11.8 & 16.1 & 15.9 & 19.3 & 28.3 & 15.2 & 14.6 \\
\hline$\Delta H_{\mathrm{f}}$ & 11.8 & 20.0 & 14.1 & 23.4 & 52.6 & 15.1 & 14.7 \\
\hline IE & 13.8 & 15.2 & 18.8 & 18.8 & 16.1 & 17.8 & 17.0 \\
\hline EA & 10.0 & 13.0 & 15.7 & 15.2 & 10.4 & 12.8 & 12.1 \\
\hline \multirow[t]{2}{*}{ PA } & 4.0 & 4.9 & 5.5 & 4.7 & 7.1 & 5.6 & 4.3 \\
\hline & \multicolumn{7}{|c|}{ E1 Subset } \\
\hline All & 11.8 & 20.7 & 17.5 & 28.1 & 57.1 & 14.2 & 25.0 \\
\hline G3/99' & 15.2 & 29.7 & 24.7 & 42.2 & 92.8 & 19.8 & 36.2 \\
\hline Add & 4.3 & 7.6 & 8.7 & 12.6 & 9.2 & 4.6 & 13.9 \\
\hline Abs & 10.4 & 11.2 & 7.4 & 7.7 & 13.0 & 8.8 & 8.9 \\
\hline \multirow[t]{2}{*}{ PR8 } & 4.2 & 6.7 & 9.4 & 11.1 & 10.6 & 7.7 & 7.6 \\
\hline & \multicolumn{7}{|c|}{ DSD $^{\prime}$ Subset } \\
\hline All & 17.5 & 20.8 & 27.8 & 29.9 & 22.5 & 24.6 & 26.9 \\
\hline S22 & 7.8 & 8.2 & 12.5 & 9.3 & 5.5 & 16.3 & 14.9 \\
\hline MB08 & 20.5 & 24.8 & 33.5 & 37.0 & 27.9 & 28.1 & 30.3 \\
\hline $\mathrm{Pd}$ & 5.2 & 5.8 & 2.7 & 2.1 & 2.7 & 4.7 & 6.3 \\
\hline Grubbs & 13.5 & 10.9 & 15.8 & 12.4 & 9.2 & 27.5 & 46.7 \\
\hline
\end{tabular}

${ }^{a}$ See ref 19 for the description of the E3 test set and its subsets.

somewhat more solid ground than its immediate predecessors. The successor to M08-type procedures, namely M11-type functionals, further makes use of range-separation. However, as we have seen earlier (Table 1), there is a deteriorated performance for M11-type methods for the CEPX33 set. Such an observation is in accord with the often-poorer results for this set for range-separated procedures when compared with the corresponding global hybrid functionals. ${ }^{2}$ This further highlights the distinct difficulty in the theoretical treatment of these complexes and transition structures.

M05-type and M06-type functionals are generally accurate and robust. However, the immediate predecessor (PW6-B95) to M05-type and M06-type functionals appears to have a similar accuracy while being even more robust. Although the development of an improved functional is beyond the scope of the present study, it would be interesting to explore the possibility of an improved version of PW6-B95, perhaps with the inclusion of a $\tau$-enhancement factor in the exchange functional and a matching dispersion correction, ${ }^{45}$ but with the use of a minimal number of additional parameters.

For the time being, we note that M06-2X has achieved the lowest weighted MAD for a hybrid-DFT procedure for the diverse GMTKN30 test set $^{2}$ and in our own benchmark studies; ${ }^{9}$ we trust that it will continue to provide outstanding performance for many other systems. However, in cases where dubious results arise from M06-2X calculations, we propose the use of PW6-B95 as an initial cross-validation.

\section{CONCLUDING REMARKS}

In the present study, we have examined the performance of a variety of DFT procedures for the calculation of complexation energies and proton-exchange barriers for water, ammonia and hydrogen fluoride clusters (the CEPX33 set), paying special attention to the M05-type and M06-type functionals. The following major findings have emerged from our investigation.

1. Typical hybrid-GGA functionals perform adequately for complexation energies but do not fare as well for the corresponding proton-exchange barriers. However, several procedures, including PW6-B95, which has been shown to be one of the most robust methods for the large GMTKN30 test set, yield good agreement with our benchmark values for the CEPX33 set.

2. An increase in the proportion of Hartree-Fock exchange leads to more positive proton-exchange barriers for (hybrid)-BLYP-type procedures, with the DFT-exchange-correlation components of the total barriers generally contributing to this trend. On the other hand, the DFT-exchange-correlation contributions to the barriers as we go from M06-L to M06 and M06-2X are negative and become more negative with increasing Hartree-Fock exchange. This results in the unusual observation of an overall lowering of the proton-exchange barriers with an increasing amount of Hartree-Fock exchange for M06-type methods.

3. Proton-exchange barriers in small clusters have previously been found ${ }^{12}$ to represent a challenge also to the high-level G4(MP2) composite procedure, but the cause for this is clearly unrelated to the M06-type difficulties found here. The two observations together highlight the difficulty in the theoretical treatment of such systems.

4. Benchmarking of combinations of the MPW-type and PBE-type exchange functionals and B95-type and PBE-type correlation functionals against the extensive E3 test set points toward PW6-B95 as the most promising functional for a wide variety of thermochemical properties, as well as for complexation energies and proton-exchange barriers. This is followed by the PBE1-PBE and PBE1-B95 combinations, with the latter showing significantly poorer performance.

5. Because M05-type and M06-type procedures are based on a $\tau$-hybrid-PBEx-B97c functional form, which is itself closely related to PBE1-B95, it would be of interest to formulate and examine the general performance of a DFT method based on the seemingly better PW6-B95.

\section{ASSOCIATED CONTENT}

S Supporting Information

Electronic energies for the various DFT methods for the CEPX33 set (Table S1) and the associated deviations from benchmark values (Table S2), electronic energies for the E3 set (Table S3), and the deviations from benchmark values (Table S4). This material is available free of charge via the Internet at http://pubs.acs.org.

\section{AUTHOR INFORMATION}

\section{Corresponding Authors}

*E-mail: bun.chan@sydney.edu.au (B.C.).

*E-mail: peter.gill@anu.edu.au (P.M.W.G.).

*E-mail: radom@chem.usyd.edu.au (L.R.).

\section{Notes}

The authors declare no competing financial interest. 


\section{ACKNOWLEDGMENTS}

We thank Dr. Amir Karton and Dr. Lars Goerigk for useful discussions and Gaussian Inc. for technical advice. We acknowledge funding (to L.R.) from the Australian Research Council (ARC) and generous grants of computer time from the National Computational Infrastructure (NCI) National Facility and Intersect Australia Ltd.

\section{REFERENCES}

(1) For general overviews of density functional theory and its applications, see for example: (a) Parr, R. G.; Yang, W. DensityFunctional Theory of Atoms and Molecules; Oxford University Press: New York, 1989. (b) Koch, W.; Holthausen, M. C. A Chemist's Guide to Density Functional Theory, 2nd ed.; Wiley: New York, 2001. (c) Scuseria, G. E.; Staroverov, V. N. In Theory and Applications of Computational Chemistry: The First Forty Years; Dykstra, C. E., Frenking, G., Kim, K. S., Scuseria, G. E., Eds.; Elsevier B. V.: Amsterdam, 2005. (d) Sholl, D.; Steckel, J. A. Density Functional Theory: A Practical Introduction; Wiley: Hoboken, 2009.

(2) For a recent comprehensive benchmark study, see: Goerigk, L.; Grimme, S. Phys. Chem. Chem. Phys. 2011, 13, 6670-6688.

(3) (a) Zhao, Y.; Truhlar, D. G. J. Phys. Chem. A 2004, 108, 69086918. (b) Zhao, Y.; Truhlar, D. G. J. Phys. Chem. A 2005, 109, 56565667.

(4) (a) Zhao, Y.; Schultz, N. E.; Truhlar, D. G. J. Chem. Phys. 2005, 123, 161103-1-4. (b) Zhao, Y.; Schultz, N. E.; Truhlar, D. G. J. Chem. Theory Comput. 2006, 2, 364-382.

(5) (a) Zhao, Y.; Truhlar, D. G. J. Chem. Phys. 2006, 125, 194101-118. (b) Zhao, Y.; Truhlar, D. G. J. Phys. Chem. A 2006, 110, 1312613130. (c) Zhao, Y.; Truhlar, D. G. Theor. Chem. Acc. 2008, 120, 215241.

(6) Zhao, Y.; Truhlar, D. G. J. Chem. Theory Comput. 2008, 4, 18491868

(7) (a) Peverati, R.; Truhlar, D. G. J. Phys. Chem. Lett. 2011, 2, 2810-2817. (b) Peverati, R.; Truhlar, D. G. J. Phys. Chem. Lett. 2012, $3,117-124$

(8) Goerigk, L.; Grimme, S. J. Chem. Theory Comput. 2011, 7, 291309.

(9) (a) Chan, B.; Radom, L. Can. J. Chem. 2010, 88, 866-876. (b) Chan, B.; Morris, M.; Radom, L. Aust. J. Chem. 2011, 64, 394-402.

(c) Chan, B.; Radom, L. Theor. Chem. Acc. 2011, 130, 251-260.

(d) Chan, B.; Radom, L. J. Phys. Chem. A 2012, 116, 4975-4986.

(e) Chan, B.; Radom, L. J. Phys. Chem. A 2013, 117, 3666-3675.

(10) (a) Jimenez-Hoyos, C. A.; Janesko, B. G.; Scuseria, G. E. Phys. Chem. Chem. Phys. 2008, 10, 6621-6629. (b) Johnson, E. R.; Becke, A. D.; Sherrill, C. D.; DiLabio, G. A. J. Chem. Phys. 2009, 131, 034111-17.

(11) Goerigk, L.; Kruse, H.; Grimme, S. ChemPhysChem 2011, 12, $3421-3433$

(12) Mardirossian, N.; Head-Gordon, M. J. Chem. Theory Comput. 2013, 9, 4453-4461.

(13) (a) Curtiss, L. A.; Redfern, P. C.; Raghavachari, K. J. Chem. Phys. 2007, 127, 124105-1-8. (b) Chan, B.; Deng, J.; Radom, L. J. Chem. Theory Comput. 2011, 7, 112-120.

(14) Curtiss, L. A.; Redfern, P. C.; Raghavachari, K. J. Chem. Phys. 2007, 126, 084108-1-12.

(15) Karton, A.; O'Reilly, R. J.; Chan, B.; Radom, L. J. Chem. Theory Comput. 2012, 8, 3128-3136.

(16) Krylov, A. I.; Gill, P. M. W. WIREs Comput. Mol. Sci. 2013, 3, 317-326.

(17) Frisch, M. J.; Trucks, G. W.; Schlegel, H. B.; Scuseria, G. E.; Robb, M. A.; Cheeseman, J. R.; Scalmani, G.; Barone, V.; Mennucci, B.; Petersson, G. A.; Nakatsuji, H.; Caricato, M.; Li, X.; Hratchian, H. P.; Izmaylov, A. F.; Bloino, J.; Zheng, G.; Sonnenberg, J. L.; Hada, M.; Ehara, M.; Toyota, K.; Fukuda, R.; Hasegawa, J.; Ishida, M.; Nakajima, T.; Honda, Y.; Kitao, O.; Nakai, H.; Vreven, T.; Montgomery, J. A., Jr.; Peralta, J. E.; Ogliaro, F.; Bearpark, M.; Heyd, J. J.; Brothers, E.; Kudin, K. N.; Staroverov, V. N.; Kobayashi, R.; Normand, J.; Raghavachari, K.;
Rendell, A.; Burant, J. C.; Iyengar, S. S.; Tomasi, J.; Cossi, M.; Rega, N.; Millam, N. J.; Klene, M.; Knox, J. E.; Cross, J. B.; Bakken, V.; Adamo, C.; Jaramillo, J.; Gomperts, R. E.; Stratmann, O.; Yazyev, A. J.; Austin, R; Cammi, C.; Pomelli, J. W.; Ochterski, R.; Martin, R. L.; Morokuma, K.; Zakrzewski, V. G.; Voth, G. A.; Salvador, P.; Dannenberg, J. J.; Dapprich, S.; Daniels, A. D.; Farkas, O.; Foresman, J. B.; Ortiz, J. V.; Cioslowski, J.; Fox, D. J. Gaussian 09, Revision C.01; Gaussian, Inc.: Wallingford CT, 2009.

(18) Neese, F. WIREs Comput. Mol. Sci. 2012, 2, 73-78.

(19) Chan, B.; Radom, L. J. Chem. Theory. Comput. 2011, 7, 28522863.

(20) (a) Curtiss, L. A.; Raghavachari, K.; Redfern, P. C.; Pople, J. A. J. Chem. Phys. 1997, 106, 1063-1079. (b) Curtiss, L. A.; Redfern, P. C.; Raghavachari, K.; Pople, J. A. J. Chem. Phys. 1998, 109, 42-55.

(21) Chan, B.; Gill, P. M. W.; Radom, L. J. Chem. Theory. Comput. 2012, 8, 4899-4906.

(22) Obtained from the EMSL Basis Set Exchange: (a) Feller, D. J. Comput. Chem. 1996, 17, 1571-1586. (b) Schuchardt, K. L.; Didier, B. T.; Elsethagen, T.; Sun, L.; Gurumoorthi, V.; Chase, J.; Li, J.; Windus, T. L. J. Chem. Inf. Model. 2007, 47, 1045-1052. (c) https://bse.pnl. gov/bse/portal (accessed March 2010).

(23) Perdew, J. P.; Burke, K.; Ernzerhof, M. Phys. Rev. Lett. 1996, 77, 3865-3868.

(24) Becke, A. D. J. Chem. Phys. 1997, 107, 8554-8560.

(25) Van Voorhis, T.; Scuseria, G. E. J. Chem. Phys. 1998, 109, 400410.

(26) Karton, A.; Martin, J. M. L. J. Chem. Phys. 2012, 136, 124114-112.

(27) Johnson, B. G.; Gill, P. M. W.; Pople, J. A. J. Chem. Phys. 1993, $98,5612-5626$

(28) The B95 correlation functional ${ }^{29}$ is the predecessor of the correlation functional in $B 97^{24}$ (i.e., B97c), with a major difference being that the two correlation terms in B97c are each expressed as a polynomial with linear adjustable parameters fitted to a training set.

(29) Becke, A. D. J. Chem. Phys. 1996, 104, 1040-1046.

(30) Adamo, C.; Barone, V. Chem. Phys. Lett. 1997, 274, 242-250.

(31) Becke, A. D. J. Chem. Phys. 1993, 98, 1372-1377.

(32) Stephens, P. J.; Devlin, F. J.; Chabalowski, C. F.; Frisch, M. J. J. Phys. Chem. 1994, 98, 11623-11627.

(33) Yanai, T.; Tew, D.; Handy, N. Chem. Phys. Lett. 2004, 393, 5160.

(34) Adamo, C.; Barone, V. J. Chem. Phys. 1999, 110, 6158-6169.

(35) Vydrov, O. A.; Scuseria, G. E. J. Chem. Phys. 2006, 125, 234109$1-9$.

(36) Heyd, J.; Scuseria, G. E.; Ernzerhof, M. J. Chem. Phys. 2006, 124, 219906-1.

(37) Schmider, H. L.; Becke, A. D. J. Chem. Phys. 1998, 108, 96249631.

(38) Boese, A. D.; Martin, J. M. L. J. Chem. Phys. 2004, 121, 34053416.

(39) Chai, J.-D.; Head-Gordon, M. J. Chem. Phys. 2008, 128, 084106$1-15$

(40) Baker, J.; Muir, M.; Audzelm, J.; Scheimner, A. ACS Symp. Ser. 1996, 629, 342-367.

(41) Schultz, N. E.; Zhao, Y.; Truhlar, D. G. J. Phys. Chem. A 2005, 109, 11127-11143.

(42) Nachimuthu, S.; Gao, J.; Truhlar, D. G. Chem. Phys. 2012, 400, $8-12$.

(43) Chan, B.; Radom, L. J. Chem. Theory Comput. 2012, 8, 42594269.

(44) We have also examined PBE-PBE-type procedures with 0,25 , and $50 \% \mathrm{HF}$ and find that they behave very similarly to the B-LYP-type methods.

(45) (a) Grimme, S.; Antony, J.; Ehrlich, S.; Krieg, H. J. Chem. Phys. 2010, 132, 154104-1-19. (b) Grimme, S.; Ehrlich, S.; Goerigk, L. J. Comput. Chem. 2011, 32, 1456-1465. 